\title{
Strong decays of the explicitly exotic doubly charmed $D D K$ bound state
}

\author{
Yin Huang $\odot$, Ming-Zhu Liu, and Ya-Wen Pan \\ School of Physics, Beihang University, Beijing 100191, China \\ Li-Sheng Geng $\oplus^{*}$ \\ School of Physics and Beijing Advanced Innovation Center for Big Data-based Precision Medicine, \\ Beihang University, Beijing 100191, China \\ and School of Physics, Zhengzhou University, Zhengzhou, Henan 450001, China
}

A. Martínez Torres $\oplus^{\dagger}$

Instituto de Física, Universidade de São Paulo, C.P. 66318, 05389-970 São Paulo, São Paulo, Brazil and School of Physics and Nuclear Energy Engineering and Beijing Key Laboratory of Advanced Nuclear Materials and Physics, Beihang University, Beijing 100191, China

\author{
K. P. Khemchandani ${ }^{\ddagger}$ \\ Universidade Federal de São Paulo, C.P. 01302-907, São Paulo, Brazil \\ and School of Physics and Nuclear Energy Engineering \\ and Beijing Key Laboratory of Advanced Nuclear Materials and Physics, \\ Beihang University, Beijing 100191, China
}

(Received 23 September 2019; published 28 January 2020)

\begin{abstract}
Nowadays, it is generally accepted that the $D K$ interaction in isospin zero is strongly attractive, and the $D_{s 0}^{*}(2317)$ can be described as a $D K$ molecular state. Recent studies have shown that the three-body $D D K$ system binds as well with a binding energy about $60-70 \mathrm{MeV}$. The $D D K$ bound state has an isospin of $1 / 2$ and a spin-parity of $0^{-}$. If discovered either experimentally or in lattice QCD, it will not only provide further support for the molecular nature of the $D_{s 0}^{*}(2317)$, but also provide a way to understand other exotic hadrons expected to be of a molecular nature. In the present work, we study its two-body strong decay widths via triangle diagrams. We find that the partial decay width into $D D_{s} \pi$ is at the order of 2-3 MeV, which seems to be within the reach of the current experiments such as Belle II. As a result, we strongly recommend that this decay channel of the $D D K$ bound state is searched for experimentally.
\end{abstract}

DOI: $10.1103 /$ PhysRevD.101.014022

\section{INTRODUCTION}

In 2003, the BABAR Collaboration observed a narrow state in the inclusive $D_{s}^{+} \pi^{0}$ invariant mass distribution of the $e^{+} e^{-}$collision at energies near $10.6 \mathrm{GeV}$ [1], i.e., the $D_{s 0}^{*}(2317)\left(D_{s 0}\right.$ for short in the present work), which was later confirmed by the CLEO Collaboration [2] and the Belle Collaboration [3]. Because the low mass, small width, and decay mode of the $D_{s 0}$ are quite different from those of a conventional $J^{P}=0^{+} c \bar{s}$ state in the naive quark model, its

\footnotetext{
*lisheng.geng@buaa.edu.cn

†amartine@if.usp.br

${ }^{*}$ kanchan.khemchandani@unifesp.br
}

Published by the American Physical Society under the terms of the Creative Commons Attribution 4.0 International license. Further distribution of this work must maintain attribution to the author(s) and the published article's title, journal citation, and DOI. Funded by SCOAP . nature has remained a topic of tremendous theoretical interest ever since its discovery [4-37]. In recent years, the importance of the $D K$ interaction in forming the $D_{s 0}$ has been confirmed by lattice QCD simulations [38-42]. See Ref. [43] for a short summary of the theoretical, experimental, and lattice QCD supports for the molecular interpretation of the $D_{s 0}$ as a $D K$ bound state.

If the $D_{s 0}$ is indeed (dominantly) a $D K$ bound state, a natural question to ask is whether the $D D K$ three-body system is still bound. In Ref. [44], by describing the $D_{s 0} D$ interaction via a one kaon exchange (OKE), it was shown that the OKE interaction is strong enough to form a $D_{s 0} D$ molecular state with a binding energy of $50-60 \mathrm{MeV}$, regardless whether the $D_{s 0} D K$ coupling is determined by treating the $D_{s 0}$ as a $c \bar{s}$ state or a $D K$ molecule. In Ref. [45], a study was done by explicitly considering the three-body $D\left(D K-D_{s} \pi-D_{s} \eta\right)$ system and by solving the Faddeev equations using the two-body inputs provided by the unitarized chiral perturbation theory and the local hidden 
symmetry approach. A three-body bound state was found in this latter work, with a total mass around $4140 \mathrm{MeV}$, which is an isospin doublet containing two states $\left(R^{++}, R^{+}\right)$. In a more recent work [46], using the Gaussian expansion method, the existence of this state has been further confirmed though with a slightly smaller binding energy of $\sim 60-70 \mathrm{MeV}$, and it has been found that even the $D D D K$ or $D D D_{s 0}$ system is bound. It is interesting to note that the $D \bar{D}^{*} K$ [47-49], the $D K K$ and $D K \bar{K}$ [50], as well as the $D K \bar{N}$ [51] systems bind as well because of the strong attraction between $D$ and $K$.

As pointed out in Ref. [45], the three-body $D D K$ bound state can decay strongly via diagrams such as those shown in Fig. 1. In the present work, we calculate explicitly the partial decay widths from such processes, aiming to provide further motivation for the experimental search for this state. The present manuscript is organized as follows. The theoretical formalism is explained in Sec. II. The predicted partial decay widths are presented in Sec. III, followed by a short summary in Sec. IV.

\section{THEORETICAL FORMALISM}

In the following, we focus on the doubly charged state $R^{++}$. Due to isospin symmetry, the decay width of its isospin partner $R^{+}$can be calculated analogously, and only small differences are expected because of the slightly different masses of its molecular components. As mentioned in Ref. [45], though the $R^{++}$is a bound state of the $D D K$ system or the $D_{s 0} \mathrm{D}$ system, it is possible for such a state to decay strongly. Keeping in mind that the $D_{s 0}^{+}$is observed in the inclusive $D_{s}^{+} \pi^{0}$ invariant mass distribution, which violates isospin, the $R^{++}$can decay via $R^{++} \equiv$ $D_{s 0}^{+} D^{+} \rightarrow\left(D_{s}^{+} \pi^{0}\right) D^{+}$. An alternative process, without involving isospin breaking, is via triangle diagrams such as those shown in Fig. 1. These processes conserve isospin

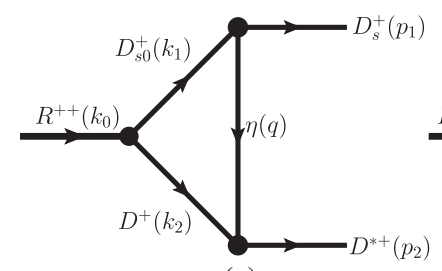

(a)

(c)

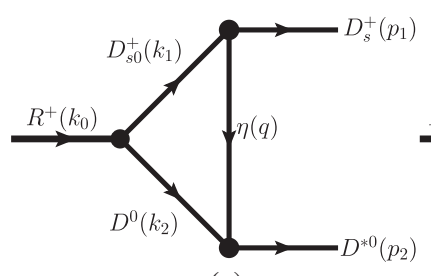

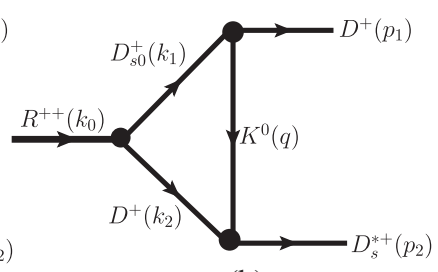

(b)

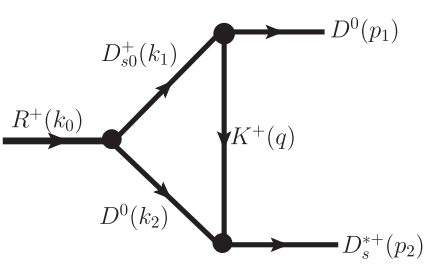

(d)
FIG. 1. Triangle diagrams representing the decay of the $R^{++}$ state to $D_{s}^{+} D^{*+}$ and $D^{+} D_{s}^{*+}$ (a)-(b), and $R^{+}$state to $D_{s}^{+} D^{* 0}$ and $D^{0} D_{s}^{*+}(\mathrm{c})-(\mathrm{d})$. and therefore, should be the dominant ones, as compared to the ones that violate isospin.

It should be noted that here we consider only $\eta$ and $K$ exchanges because $D K$ and $D_{s} \eta$ are the only two relevant channels in the dynamical generations of the $D_{s 0}^{*}(2317)$ (see, e.g., Refs. [38-42] and references cited therein). The replacement of the $\eta / K$ by $\phi / K^{*}$ is not allowed by angular momentum and parity conservations. In addition, the $R^{++/+}$can not decay into a baryon-antibaryon pair such as $\Xi_{c c}^{++} \bar{\Lambda}$ because there is no phase space for such decays. In principle, one may wonder if the $D^{*} D_{s}$ and $D D_{s}^{*}$ play a role in the dynamical generation of the $R^{++/+}$, but they do not. This can be understood from two perspectives. First, the $R^{++/+}$state is dynamically generated from $D(D K) \sim$ $D D_{s 0}^{*}(2317)$ interactions. Second, the thresholds of $D^{*} D_{s}$ and $D D_{s}^{*}$ are about $160 \mathrm{MeV}$ below the pole position of the $R^{++/+}$, which further implies that these two channels play a marginal role in the dynamical generation of the $R^{++/+}$.

In the following, we explain how to calculate the four diagrams shown in Fig. 1.

In order to calculate the Feynman diagrams shown in Fig. 1, we need to determine the relevant vertices. For the vertex of $R^{++} D_{s 0}^{+} D^{+}$, since the $R^{++}$can be treated as a bound state of $D_{s 0}^{+} D^{+}$[44], this coupling can be determined by the Weinberg compositeness condition. In the present work, we adopt the method developed in Refs. [52-68]. In this framework, the interacting Lagrangian between $R, D_{s 0}$, and $D$ can be written as [52,53]

$$
\begin{aligned}
\mathcal{L}_{R}(x)= & g_{R D_{s 0} D}(x) R^{T}(x) \int d y \Phi_{R}\left(y^{2}\right) D_{s 0}\left(x+\omega_{D} y\right) \\
& \times D\left(x-\omega_{D_{s 0}} y\right)+\text { H.c. },
\end{aligned}
$$

where $\omega_{i}=m_{i} /\left(m_{i}+m_{j}\right)$ is a kinematical parameter with $m_{i}$ and $m_{j}$ being the masses of the involved mesons. In the Lagrangian of Eq. (1), an effective correlation function $\Phi\left(y^{2}\right)$ is introduced to reflect the distribution of the two constituents, $D_{s 0}^{+}(2317)$ and $D^{+}\left(D^{0}\right)$, in the hadronic molecular $R^{++}\left(R^{+}\right)$state. The introduced correlation function also serves the purpose of making the Feynman diagrams ultraviolet finite. Here, we choose the Fourier transformation of the correlation function in terms of a Gaussian form,

$$
\Phi\left(p^{2}\right) \doteq \exp \left(-p_{E}^{2} / \Lambda^{2}\right),
$$

where $\Lambda \sim 1.0 \mathrm{GeV}$ [52-68] is a size parameter, which characterizes the distribution of the molecular components inside the molecule, and $p_{E}$ is the Euclidean Jacobi momentum [52-68]. In the present work, we take $\Lambda=1 \mathrm{GeV}$, unless otherwise stated.

The coupling constant $g_{R D_{50} D}$ in Eq. (1) could be determined by the compositeness condition [52,53], where the renormalization constant of the composite particle should be zero, i.e., 


$$
Z_{R} \equiv 1-\Sigma_{R}\left(m_{R}^{2}\right)=0,
$$

with $\Sigma_{R}\left(m_{R}^{2}\right)$ being the derivative of the mass operator of the $R$. The concrete form of the mass operator of the $D D K$ bound state $R$ corresponding to the diagram in Fig. 2 is

$$
\begin{aligned}
\mathcal{W}_{R}\left(k_{0}\right)= & \frac{g_{R D_{s 0} D}^{2}}{16 \pi^{2}} \int_{0}^{\infty} d \alpha \int_{0}^{\infty} d \beta \frac{1}{z^{2}} \exp \left\{-\frac{1}{\Lambda^{2}}\right. \\
& \left.\times\left[-2 k_{0}^{2} \omega_{D_{s 0}}^{2}+\alpha m_{D_{s 0}}^{2}+\beta\left(-k_{0}^{2}+m_{D}^{2}\right)+\frac{\Delta_{M}^{2}}{4 z}\right]\right\},
\end{aligned}
$$

where $z=2+\alpha+\beta, \Delta_{M}=-4 \omega_{D_{s 0}} k_{0}-2 \beta k_{0}$, and $k_{0}^{2}=m_{R}^{2}$ with $k_{0}, m_{R}$ denoting the four momenta and mass of the $R$, respectively. Here, we set $m_{R}=m_{D_{s 0}}+m_{D}-E_{b}$ with $E_{b}$ being the binding energy of $R, k_{1}$ and $m_{D_{50}}$ are the four momenta and mass of the $D_{s 0}$, and $m_{D}$ is the mass of the $D$ meson, respectively.

In the present work, we calculate the two-body decay width of the $R$ via the triangle diagrams shown in Fig. 1. To evaluate the diagrams, in addition to the Lagrangian of Eq. (1), the following effective Lagrangian terms, responsible for the interactions between heavy-light pseudoscalar and vector mesons, are needed as well [33]:

$$
\mathcal{L}_{P \phi P^{*}}=i g\left\langle P_{\mu}^{*} u^{\mu} P^{\dagger}-P u^{\mu} P_{\mu}^{* \dagger}\right\rangle,
$$

where $P=\left(D^{0}, D^{+}, D_{s}^{+}\right)$and $P^{*}=\left(D^{* 0}, D^{*+}, D_{s}^{*+}\right), u^{\mu}$ is the axial vector combination of the pseudoscalar-meson fields and their derivatives,

$$
u^{\mu}=i\left(u^{\dagger} \partial^{\mu} u-u \partial^{\mu} u^{\dagger}\right)
$$

where $u^{2}=U=\exp \left(i \frac{\phi}{f_{0}}\right), \quad f_{0}=92.4 \mathrm{MeV}$, and the pseudoscalar- meson octet $\phi$ is represented by the $3 \times 3$ matrix,

$$
\phi=\sqrt{2}\left(\begin{array}{ccc}
\frac{\pi^{0}}{\sqrt{2}}+\frac{\eta}{\sqrt{6}} & \pi^{+} & K^{+} \\
\pi^{-} & -\frac{\pi^{0}}{\sqrt{2}}+\frac{\eta}{\sqrt{6}} & K^{0} \\
K^{-} & \bar{K}^{0} & -\frac{2}{\sqrt{6}} \eta
\end{array}\right)
$$

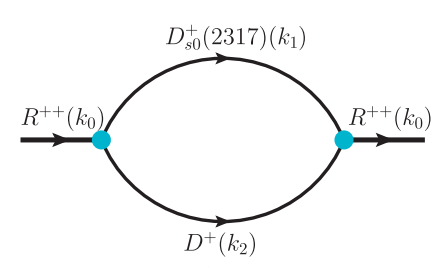

(a)

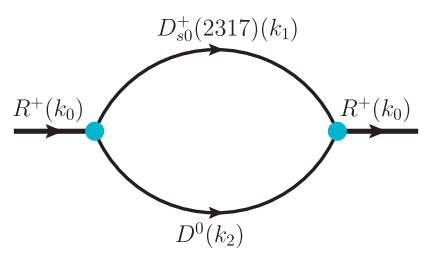

(b)
FIG. 2. Self-energy of the $R^{++}$and $R^{+}$states.
From Eqs. (5)-(7), one can easily obtain the interaction vertices $\eta D D^{*}, K D D_{s}^{*}$, and $\pi D^{*} D$. The coupling constant $g$ can be determined from the strong decay width $\Gamma\left(D^{*+} \rightarrow D^{0} \pi^{+}\right)=56.46 \pm 1.22 \mathrm{keV}$, together with the branching ratio $\operatorname{BR}\left(D^{*+} \rightarrow D^{0} \pi^{+}\right)=(67.7 \pm 0.5) \%$ [69]. With the help of Eq. (5), the two body decay width $\Gamma\left(D^{*+} \rightarrow \pi^{+} D^{0}\right)$ is related to $g$ via

$$
\Gamma\left(D^{*+} \rightarrow \pi^{+} D^{0}\right)=\frac{1}{12 \pi} \frac{g^{2}}{f_{0}^{2}} \frac{\left|\vec{p}_{\pi}\right|^{3}}{M^{2}},
$$

where $\vec{p}_{\pi}$ is the three momentum of $\pi^{+}$in the rest frame of the decaying vector meson $D^{*+}$. Using the corresponding experimental strong decay width and the masses of the relevant particles given in Table I [69], we obtain $g=1.097 \pm 0.012 \mathrm{GeV}$.

In the chiral unitary approaches $[20,21,33,70]$, the $D_{s 0}$ is found to be dynamically generated from the $D K$ and $D_{s} \eta S$-wave interactions. As a result, the vertices $D_{s 0} D K$ and $D_{s 0} \eta D_{s}$ can be easily written as

$$
\begin{aligned}
& \mathcal{L}_{D_{s 0} D K}=g_{D_{s 0} D K} D_{s 0} D K, \\
& \mathcal{L}_{D_{s 0} D_{s} \eta}=g_{D_{s 0} D_{s} \eta} D_{s 0} D_{s} \eta,
\end{aligned}
$$

where the coupling of the $D_{s 0}$ to $D K$ and $D_{s} \eta$ states, $g_{D_{s 0} D K}$ and $g_{D_{s 0} D_{s} \eta}$, can be obtained from the coupling constant of the $D_{s 0}$ to the $D K$ and $\eta D_{s}$ channels in isospin zero, which are found to be $g_{D_{s 0} D K}=10.21 \mathrm{GeV}(10.203 \mathrm{GeV})$ and $g_{D_{s 0} D_{s} \eta}=6.40 \mathrm{GeV}$ (5.876 GeV) in Ref. [21] ([20]), multiplied by the appropriate Clebsch-Gordan (CG) coefficients, namely, $g_{D_{s 0}^{+} D^{+} K^{0}}=g_{D_{s 0}^{+} D^{0} K^{+}}=-g_{D_{s 0} D K} / \sqrt{2}$ and $g_{D_{s 0}^{+} D_{s}^{+} \eta}=g_{D_{s 0} D_{s} \eta}$.

With the above vertices, the amplitudes of the triangle diagrams of Fig. 1, evaluated in the center of mass frame of final states, are

$$
\begin{aligned}
-i \mathcal{M}_{\eta}^{a}= & g_{R^{++} D_{s 0}^{+} D^{+}} g_{D_{s 0}^{+} D_{s}^{+} \eta} \frac{-g}{\sqrt{3} f_{0}} \int \frac{d^{4} q}{(2 \pi)^{4}} \Phi\left[\left(k_{1} \omega_{D^{+}}\right.\right. \\
& \left.\left.-k_{2} \omega_{D_{s 0}^{+}}\right)^{2}\right] \epsilon_{\mu}\left(p_{2}\right) q^{\mu} \frac{1}{q^{2}-m_{\eta}^{2}} \frac{1}{k_{1}^{2}-m_{D_{s 0}^{+}}^{2}} \frac{1}{k_{2}^{2}-m_{D^{+}}^{2}},
\end{aligned}
$$

TABLE I. Masses of the relevant particles in the present work (in units of MeV) [69].

\begin{tabular}{lcccc}
\hline \hline$D^{+}$ & $D^{0}$ & $\eta$ & $D^{* 0}$ & $D^{* \pm}$ \\
\hline 1869.65 & 1864.83 & 547.862 & 2006.85 & 2010.26 \\
\hline \hline$K^{0}$ & $D_{s}^{* \pm}$ & $D_{s 0}^{* \pm}$ & $D_{s}^{ \pm}$ & $K^{ \pm}$ \\
\hline 497.611 & 2112.2 & 2317.0 & 1968.34 & 493.677 \\
\hline \hline
\end{tabular}




$$
\begin{aligned}
-i \mathcal{M}_{K^{0}}^{b}= & g_{R^{++} D_{s 0}^{+} D^{+}} g_{D_{s 0}^{+} D^{+} K^{0}} \\
& \times \frac{-\sqrt{2} g}{f_{0}} \int \frac{d^{4} q}{(2 \pi)^{4}} \Phi\left[\left(k_{1} \omega_{D^{+}}-k_{2} \omega_{D_{s 0}^{+}}\right)^{2}\right] \\
& \times \epsilon_{\mu}\left(p_{2}\right) q^{\mu} \frac{1}{q^{2}-m_{K^{0}}^{2}} \frac{1}{k_{1}^{2}-m_{D_{s 0}^{+}}^{2}} \frac{1}{k_{2}^{2}-m_{D^{+}}^{2}}
\end{aligned}
$$

$$
\begin{aligned}
-i \mathcal{M}_{\eta}^{c}= & g_{R^{++} D_{s 0}^{+} D^{+}} g_{D_{s 0}^{+} D_{s}^{+} \eta} \\
& \times \frac{-g}{\sqrt{3} f_{0}} \int \frac{d^{4} q}{(2 \pi)^{4}} \Phi\left[\left(k_{1} \omega_{D^{0}}-k_{2} \omega_{D_{s 0}^{+}}\right)^{2}\right] \\
& \times \epsilon_{\mu}\left(p_{2}\right) q^{\mu} \frac{1}{q^{2}-m_{\eta}^{2}} \frac{1}{k_{1}^{2}-m_{D_{s 0}^{+}}^{2}} \frac{1}{k_{2}^{2}-m_{D^{0}}^{2}},
\end{aligned}
$$

$$
\begin{aligned}
-i \mathcal{M}_{K^{+}}^{d}= & g_{R^{++} D_{s 0}^{+} D^{+}} g_{D_{s 0}^{+} D^{0} K^{+}} \\
& \times \frac{-\sqrt{2} g}{f_{0}} \int \frac{d^{4} q}{(2 \pi)^{4}} \Phi\left[\left(k_{1} \omega_{D^{+}}-k_{2} \omega_{D_{s 0}^{+}}\right)^{2}\right] \\
& \times \epsilon_{\mu}\left(p_{2}\right) q^{\mu} \frac{1}{q^{2}-m_{K^{+}}^{2}} \frac{1}{k_{1}^{2}-m_{D_{s 0}^{+}}^{2}} \frac{1}{k_{2}^{2}-m_{D^{0}}^{2}} .
\end{aligned}
$$

The corresponding partial decay width then reads

$$
d \Gamma[R \rightarrow]=\frac{1}{2 J+1} \frac{1}{32 \pi^{2}} \frac{\left|\vec{p}_{1}\right|}{m_{R}^{2}} \mid \overline{\left.\mathcal{M}\right|^{2}} d \Omega,
$$

where $J=0$ is the total angular momentum of the initial $R$ state, the overline indicates the sum over the polarization vectors of final hadrons, and $\left|\vec{p}_{1}\right|$ is the three momentum of the decay products in the rest frame of the $\left(R^{++}, R^{+}\right)$states. Then the total decay widths of the $\left(R^{++}, R^{+}\right)$states are

$$
\begin{aligned}
\Gamma_{R^{++}} & =\Gamma\left[R^{++} \rightarrow D_{s}^{+} D^{*+}\right]+\Gamma\left[R^{++} \rightarrow D^{+} D_{s}^{*+}\right], \\
\Gamma_{R^{+}} & =\Gamma\left[R^{+} \rightarrow D_{s}^{+} D^{* 0}\right]+\Gamma\left[R^{+} \rightarrow D^{0} D_{s}^{*+}\right] .
\end{aligned}
$$

\section{RESULTS AND DISCUSSIONS}

To estimate the partial decay widths of the $R$, we first need to determine the coupling constants related to the molecular state and its components.

In Refs. [44-46], the $R^{++}$state is found to have a binding energy about $15-45 \mathrm{MeV}$, with respect to the $D_{s 0}^{+} D^{+}$ threshold. In this mass range, the coupling constant is dependent on the mass of the bound state $R$ as shown in Fig. 3. One finds that the coupling constant $g_{R^{++} D^{+} D_{s 0}^{+}}$ decreases with $m_{R^{++}}$. With a value of the mass $m_{R^{++}}=$ $4140 \mathrm{MeV}$, the corresponding coupling constant is $g_{R^{++} D^{+} D_{s 0}^{+}}=9.02 \mathrm{GeV}$.

We show the dependence of the total decay width on the masses of the bound state $R^{++}$in Fig. 4. One can see that the total decay width increases slightly with the mass of the

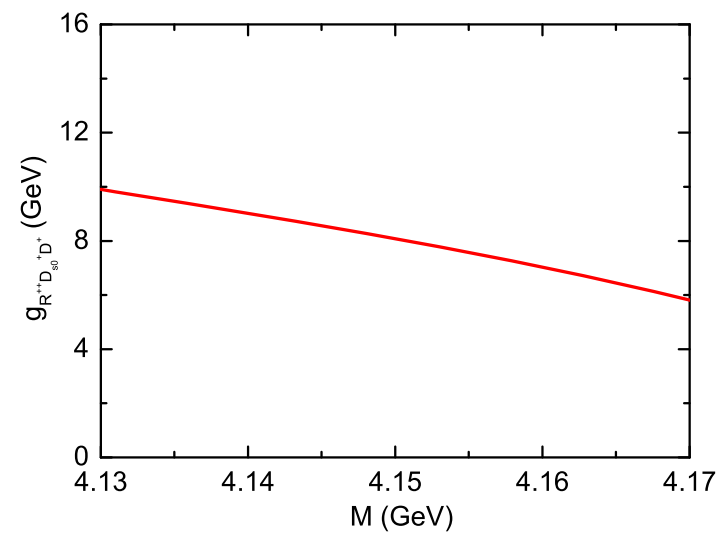

FIG. 3. The coupling constant $g_{R^{++} D_{s 0}^{+} D^{+}}$as a function of the mass of $R^{++}$.

bound state $R^{++}$from 4.13 to $4.17 \mathrm{GeV}$. The predicted total decay width is small and found to be $\Gamma_{R^{++}}=2.5-2.6 \mathrm{MeV}$.

In Fig. 4, we also show the partial decay widths of the $R^{++} \rightarrow D^{+} D_{s}^{*+}$ and $D_{s}^{+} D^{*+}$ as a function of the mass of the bound state $R$. The corresponding partial decay widths for several masses of the bound state are listed in Table II. We note that the transition $R^{++} \rightarrow D^{+} D_{s}^{*+}$ is the main decay channel, almost saturating the total width. The corresponding partial decay widths are $\Gamma_{R^{++} \rightarrow D^{+} D_{s}^{*+}}=$ 2.30-2.50 MeV and $\Gamma_{R^{++} \rightarrow D_{s}^{+} D^{*+}}=0.26-0.29 \mathrm{MeV}$, which yields a total decay width of $2.6-2.8 \mathrm{MeV}$. We note that the results depend only moderately on the cutoff. For instance, varying the cutoff from 0.5 to $1.5 \mathrm{GeV}$, the total decay width changes from 1.6 to $3.4 \mathrm{MeV}$ within the $R^{++}$mass range of 4.13 to $4.17 \mathrm{GeV}$.

We find that the contribution from the $\eta$ meson exchange is very small, because the $\eta D^{0} D^{* 0}$ vertex, which involves the creation or annihilation of an additional $s \bar{s}$ quark pair, is

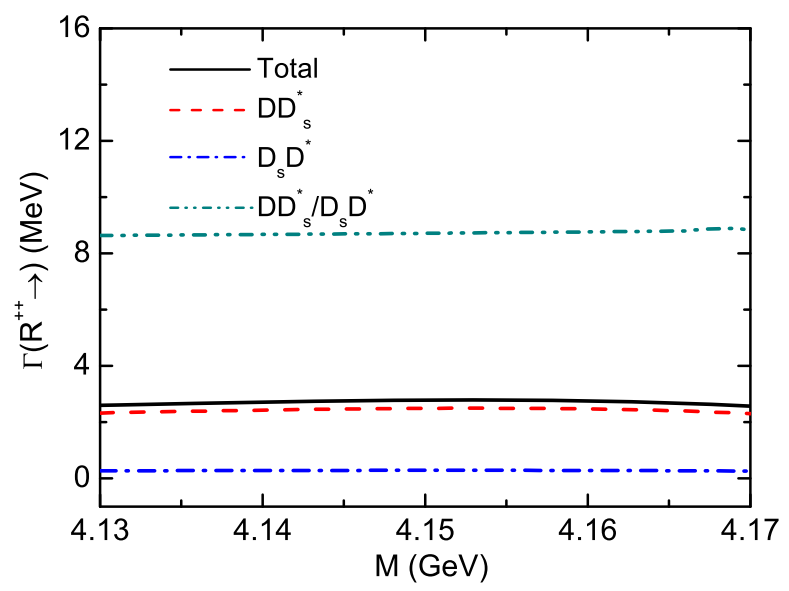

FIG. 4. Total decay width (black solid line), partial decay widths of the $R^{++} \rightarrow D^{+} D_{s}^{*+}$ (red dashed line), $R^{++} \rightarrow D_{s}^{+} D^{*+}$ (blue dash-dot-dotted line), and the ratio of the partial decay widths (dark cyan dash dotted line) as a function of the mass of the $R^{++}$. 
TABLE II. Partial decay widths of $R^{++} \rightarrow D_{s}^{+} D^{*+}$ and $D^{+} D_{s}^{*+}$ for different $R^{++}$masses (in units of $\mathrm{MeV}$ ).

\begin{tabular}{lcccccc}
\hline \hline Mode & $\Lambda(\mathrm{GeV})$ & 4130 & 4140 & 4150 & 4160 & 4170 \\
\hline$R^{++} \rightarrow D_{s}^{+} D^{*+}$ & 1.0 & 0.27 & 0.28 & 0.29 & 0.29 & 0.26 \\
$R^{++} \rightarrow D^{+} D_{s}^{*+}$ & 1.0 & 2.33 & 2.43 & 2.49 & 2.47 & 2.30 \\
\hline \hline
\end{tabular}

strongly suppressed. Moreover, the main component of the $D_{s 0}(2317)$ is $D K[20,21,33,70]$, and the coupling constant related to this vertex is larger than the others. These two factors make the contribution from the $K$ meson exchange the most important one.

In Fig. 4, we also show the ratio of the partial decay widths into $D^{+} D_{s}^{*+}$ and $D_{s}^{+} D^{*+}$. The ratio of branching fractions is found to be of the order of 8-9 and is almost independent of the mass of the bound state $R$.

\section{SUMMARY}

In this work, inspired by the recent series of studies that showed the likely existence of a $D D K$ bound state, we have studied its partial decay widths into $D_{s} D^{*}$ and $D D_{s}^{*}$. Such a decay involves the treatment of the $R$ state as a quasibound state of $D_{s 0}^{*}(2317) D$ and utilizing the Weinberg compositeness condition to determine the corresponding coupling. Our studies find a relative small total decay width, of the order of 2-3 MeV, mainly to $D D_{s}^{*}$, and the results depend only moderately on the single parameter of the method, the cutoff $\Lambda$.

The predicted decay width seems to suggest that it is possible to observe such a state at Belle or Belle II, e.g., via the inclusive invariant mass distribution $D^{+} D_{s}^{+} \pi^{0}$, which is quite similar to the experimental discovery of the $D_{s 0}^{*}(2317)$ by BABAR, Belle, and CLEO. On the other hand, its production yields at these experimental setups remain to be studied.

Recent lattice QCD studies of compact tetraquark states, see, e.g., Refs. [71,72], suggest that a study of the $D D K$ bound state in terms of its minimal quark content $c c \bar{q} \bar{s}$ might be within the reach of the state of the art of lattice QCD simulations, even taking explicitly into account its three-meson molecular nature [73].

\section{ACKNOWLEDGMENTS}

LSG thanks Cheng-Ping Shen and Manuel Pavon Valderrama for some stimulating discussions. This work was partly supported by the National Natural Science Foundation of China (NSFC) under Grants No. 11975041, No. 11735003, and Conselho Nacional de Desenvolvimento Científico e Tecnológico (CNPq) under Grants No. 310759/ 2016-1 and No. 311524/2016-8.
[1] B. Aubert et al. (BABAR Collaboration), Phys. Rev. Lett. 90, 242001 (2003).

[2] D. Besson et al. (CLEO Collaboration), Phys. Rev. D 68, 032002 (2003); 75, 119908(E) (2007).

[3] P. Krokovny et al. (Belle Collaboration), Phys. Rev. Lett. 91, 262002 (2003).

[4] W. A. Bardeen, E. J. Eichten, and C. T. Hill, Phys. Rev. D 68, 054024 (2003).

[5] M. A. Nowak, M. Rho, and I. Zahed, Acta Phys. Pol. B 35, 2377 (2004).

[6] E. van Beveren and G. Rupp, Phys. Rev. Lett. 91, 012003 (2003).

[7] Y.-B. Dai, C.-S. Huang, C. Liu, and S.-L. Zhu, Phys. Rev. D 68, 114011 (2003).

[8] S. Narison, Phys. Lett. B 605, 319 (2005).

[9] A. P. Szczepaniak, Phys. Lett. B 567, 23 (2003).

[10] T. E. Browder, S. Pakvasa, and A. A. Petrov, Phys. Lett. B 578, 365 (2004).

[11] T. Barnes, F. E. Close, and H. J. Lipkin, Phys. Rev. D 68, 054006 (2003).

[12] H.-Y. Cheng and W.-S. Hou, Phys. Lett. B 566, 193 (2003).

[13] Y.-Q. Chen and X.-Q. Li, Phys. Rev. Lett. 93, 232001 (2004).

[14] V. Dmitrasinovic, Phys. Rev. Lett. 94, 162002 (2005).

[15] J.-R. Zhang, Phys. Lett. B 789, 432 (2019).
[16] K. Terasaki, Phys. Rev. D 68, 011501 (2003).

[17] L. Maiani, F. Piccinini, A. Polosa, and V. Riquer, Phys. Rev. D 71, 014028 (2005).

[18] E. E. Kolomeitsev and M. F. M. Lutz, Phys. Lett. B 582, 39 (2004).

[19] J. Hofmann and M. F. M. Lutz, Nucl. Phys. A733, 142 (2004).

[20] F.-K. Guo, P.-N. Shen, H.-C. Chiang, R.-G. Ping, and B.-S. Zou, Phys. Lett. B 641, 278 (2006).

[21] D. Gamermann, E. Oset, D. Strottman, and M. J. Vicente Vacas, Phys. Rev. D 76, 074016 (2007).

[22] F.-K. Guo, C. Hanhart, S. Krewald, and U.-G. Meissner, Phys. Lett. B 666, 251 (2008).

[23] F.-K. Guo, C. Hanhart, and U.-G. Meissner, Eur. Phys. J. A 40, 171 (2009).

[24] M. Cleven, F.-K. Guo, C. Hanhart, and U.-G. Meissner, Eur. Phys. J. A 47, 19 (2011).

[25] A. Martinez Torres, L. R. Dai, C. Koren, D. Jido, and E. Oset, Phys. Rev. D 85, 014027 (2012).

[26] D.-L. Yao, M.-L. Du, F.-K. Guo, and U.-G. Meißner, J. High Energy Phys. 11 (2015) 058.

[27] Z.-H. Guo, U.-G. Meißner, and D.-L. Yao, Phys. Rev. D 92, 094008 (2015).

[28] M. Albaladejo, P. Fernandez-Soler, F.-K. Guo, and J. Nieves, Phys. Lett. B 767, 465 (2017). 
[29] M.-L. Du, F.-K. Guo, U.-G. Meißner, and D.-L. Yao, Eur. Phys. J. C 77, 728 (2017).

[30] X.-Y. Guo, Y. Heo, and M. F. M. Lutz, Phys. Rev. D 98, 014510 (2018).

[31] M. Albaladejo, P. Fernandez-Soler, J. Nieves, and P. G. Ortega, Eur. Phys. J. C 78, 722 (2018).

[32] M. Altenbuchinger and L.-S. Geng, Phys. Rev. D 89, 054008 (2014).

[33] M. Altenbuchinger, L. S. Geng, and W. Weise, Phys. Rev. D 89, 014026 (2014).

[34] L. S. Geng, N. Kaiser, J. Martin-Camalich, and W. Weise, Phys. Rev. D 82, 054022 (2010).

[35] P. Wang and X. G. Wang, Phys. Rev. D 86, 014030 (2012).

[36] Y.-R. Liu, X. Liu, and S.-L. Zhu, Phys. Rev. D 79, 094026 (2009).

[37] Z.-H. Guo, L. Liu, U.-G. Meißner, J. A. Oller, and A. Rusetsky, Eur. Phys. J. C 79, 13 (2019).

[38] L. Liu, K. Orginos, F.-K. Guo, C. Hanhart, and U.-G. Meissner, Phys. Rev. D 87, 014508 (2013).

[39] D. Mohler, C. B. Lang, L. Leskovec, S. Prelovsek, and R. M. Woloshyn, Phys. Rev. Lett. 111, 222001 (2013).

[40] C. B. Lang, L. Leskovec, D. Mohler, S. Prelovsek, and R. M. Woloshyn, Phys. Rev. D 90, 034510 (2014).

[41] A. Martínez Torres, E. Oset, S. Prelovsek, and A. Ramos, J. High Energy Phys. 05 (2015) 153.

[42] G. S. Bali, S. Collins, A. Cox, and A. Schäfer, Phys. Rev. D 96, 074501 (2017).

[43] F.-K. Guo, EPJ Web Conf. 202, 02001 (2019).

[44] M. Sanchez Sanchez, L.-S. Geng, J.-X. Lu, T. Hyodo, and M. P. Valderrama, Phys. Rev. D 98, 054001 (2018).

[45] A. Martinez Torres, K. Khemchandani, and L.-S. Geng, Phys. Rev. D 99, 076017 (2019).

[46] T.-W. Wu, M.-Z. Liu, L.-S. Geng, E. Hiyama, and M. P. Valderrama, Phys. Rev. D 100, 034029 (2019).

[47] L. Ma, Q. Wang, and U.-G. Meißner, Chin. Phys. C 43, 014102 (2019).

[48] X.-L. Ren, B. B. Malabarba, L.-S. Geng, K. P. Khemchandani, and A. Martínez Torres, Phys. Lett. B 785, 112 (2018).

[49] X.-L. Ren, B. B. Malabarba, K. P. Khemchandani, and A. Martinez Torres, J. High Energy Phys. 05 (2019) 103.

[50] V. R. Debastiani, J. M. Dias, and E. Oset, Phys. Rev. D 96, 016014 (2017).

[51] J. Yamagata-Sekihara and T. Sekihara, Phys. Rev. C 100, 015203 (2019).
[52] A. Faessler, T. Gutsche, V. E. Lyubovitskij, and Y.-L. Ma, Phys. Rev. D 76, 014005 (2007).

[53] A. Faessler, T. Gutsche, V. E. Lyubovitskij, and Y.-L. Ma, Phys. Rev. D 76, 114008 (2007).

[54] Y.-b. Dong, A. Faessler, T. Gutsche, and V. E. Lyubovitskij, Phys. Rev. D 77, 094013 (2008).

[55] Y. Dong, A. Faessler, T. Gutsche, and V. E. Lyubovitskij, J. Phys. G 38, 015001 (2011).

[56] Y. Dong, A. Faessler, T. Gutsche, S. Kovalenko, and V. E. Lyubovitskij, Phys. Rev. D 79, 094013 (2009).

[57] Y. Dong, A. Faessler, T. Gutsche, Q. Lü, and V.E. Lyubovitskij, Phys. Rev. D 96, 074027 (2017).

[58] Y. Dong, A. Faessler, T. Gutsche, and V. E. Lyubovitskij, Phys. Rev. D 90, 094001 (2014).

[59] Y. Dong, A. Faessler, T. Gutsche, and V. E. Lyubovitskij, Phys. Rev. D 90, 074032 (2014).

[60] Y. Dong, A. Faessler, T. Gutsche, and V. E. Lyubovitskij, Phys. Rev. D 89, 034018 (2014).

[61] Y. Dong, A. Faessler, T. Gutsche, and V. E. Lyubovitskij, Phys. Rev. D 88, 014030 (2013).

[62] Y. Dong, A. Faessler, T. Gutsche, and V. E. Lyubovitskij, Few Body Syst. 54, 1011 (2013).

[63] Y. Dong, A. Faessler, T. Gutsche, and V. E. Lyubovitskij, J. Phys. G 40, 015002 (2013).

[64] Y. Dong, A. Faessler, T. Gutsche, S. Kumano, and V. E. Lyubovitskij, Phys. Rev. D 83, 094005 (2011).

[65] Y. Dong, A. Faessler, T. Gutsche, S. Kumano, and V. E. Lyubovitskij, Phys. Rev. D 82, 034035 (2010).

[66] Y. Dong, A. Faessler, T. Gutsche, and V. E. Lyubovitskij, Phys. Rev. D 81, 074011 (2010).

[67] Y. Dong, A. Faessler, T. Gutsche, and V. E. Lyubovitskij, Phys. Rev. D 81, 014006 (2010).

[68] Y. Dong, A. Faessler, and V. E. Lyubovitskij, Prog. Part. Nucl. Phys. 94, 282 (2017).

[69] M. Tanabashi et al. (Particle Data Group), Phys. Rev. D 98, 030001 (2018).

[70] D. Gamermann and E. Oset, Eur. Phys. J. A 33, 119 (2007).

[71] A. Francis, R. J. Hudspith, R. Lewis, and K. Maltman, Phys. Rev. Lett. 118, 142001 (2017).

[72] L. Leskovec, S. Meinel, M. Pflaumer, and M. Wagner, Phys. Rev. D 100, 014503 (2019).

[73] B. Hörz and A. Hanlon, Phys. Rev. Lett. 123, 142002 (2019). 